\title{
STRUCTURE OF THE RED ABSORPTION BAND OF CHLOROPHYLL a IN ASPIDISTRA ELATIOR
}

\author{
J. B. THOMAS \\ Biophysical Research Group, Physical Institute of the State University, Utrecht (The Netherlands)
}

(Received September I4th, I96I)

\section{SUMMARY}

The red absorption band of chlorophyll $a$ in chloroplasts of Aspidistra elatior consists of a main maximum and a number of weak shoulders. This number may vary in chloroplast preparations from different leaves. At most, five shoulders were noticed.

Heat treatment affects the shoulders in a mutually different way.

Upon storage at $23^{\circ}$, the red absorption band changes in two regions: both the main peak and one of the shoulders around $660 \mathrm{~m} \mu$ shift to the long-wave side. As a rule, the shift of the main maximum needs $I-2 \mathrm{~h}$ for completion, while that of the shoulder is finished within 20-30 min.

Indication is obtained that the time course of the slow shift parallels that of the loss of ability to perform the HILl reaction with $0.02 M p$-benzoquinone as the oxidant.

It is concluded that, most probably, at least six forms of chlorophyll $a$ occur in Aspidistra chloroplasts. To what extent their functions may be different remains to be studied.

\section{INTRODUCTION}

Indication of the occurrence of both functionally and spectroscopically different forms of chlorophyll $a$ in vivo has been obtained by DUYSENS ${ }^{1}$ and Yocum AND BLINks². FRENCH AND YounG ${ }^{3}$ analyzed the results of HAXO AND BLINKS ${ }^{4}$ and concluded that a photosynthetically active form of chlorophyll $a$ shows maximum absorption around $670 \mathrm{~m} \mu$ while an inactive form absorbed mainly around $680 \mathrm{~m} \mu$. VOROB'Eva AND KRASNOVSKY ${ }^{5}$ also suggested the presence of an active and an inactive form with maximum absorption at 670 and $678 \mathrm{~m} \mu$, respectively.

The conception of the simultaneous occurrence of an active and an inactive form changed after EMERSON's finding ${ }^{6}$ that the excitation of two pigments, namely an "auxiliary" pigment and chlorophyll $a$, is required for obtaining maximum photosynthetic quantum yield. FRANCK ${ }^{7}$ suggested an explanation of this EMERSon effect by assuming the occurrence of chlorophyll $a$ in two states. Part of the pigment is suggested to be exposed to water with its solutes and may receive energy transferred from the auxiliary pigments, while part of it is "protected" against water by non-polar lipoid molecules. By energy transfer both types are supposed to cooperate in a single photochemical process. 
RABINOWITCH ${ }^{8}$ was the first to suggest that two types of chlorophyll in vivo may be involved in two different steps of the primary photochemical process. GovINDJEE AND RABINOWITCH ${ }^{9}$ stated that two chlorophyll $a$ forms with maximum absorption at 670 and $680 \mathrm{~m} \mu$ respectively-designated below as $\mathrm{C}_{a} 670$ and $\mathrm{C}_{a} 680$-play different photochemical roles in photosynthesis, while $R$. GovindjeE, Thomas and RabinoWITCH ${ }^{10}$ observed the same for the Hill reaction with quinone. DUYSENS, AMESZ AND KAMP" , working with the red alga Porphyridium cruentum, concluded that two photochemical systems operate in photosynthesis which are linked to different pigment complexes, namely $\mathrm{C}_{a} 680$ and part of chlorophyll $a$ connected with the phycobilins. Their function is suggested to consist of oxidation and reduction of cytochromes respectively. Evidence for two photochemical processes driven by different pigments was also presented by Losada, Whatley and ARnon ${ }^{12}$. Witt, Muller and Rum${ }_{B E R G}{ }^{13}$ suggested that $C_{a} 670$ and $C_{a} 680$ oxidize and reduce cytochromes in the light respectively.

At longer wavelengths $\mathrm{C}_{\boldsymbol{a}}$ forms were also observed and indication was obtained that these are involved in photochemical reactions. FRENCH ${ }^{14-17}$ suggested the importance of $C_{a} 695$. KoK ${ }^{18,19}$ observed and discussed the role of $C_{a 700}$, called by him: Pyoo. According to KRAsnovsky and KosobutsKaJA ${ }^{20}$, chlorophyll $a$ in vivo may. occur in both monomeric and polymeric states. BRODY ${ }^{21,22}$ obtained indication that the chlorophyll $a$ form responsible for the emission of fluorescence, at low temperature, around $720 \mathrm{~m} \mu$, and absorbing probably around $705 \mathrm{~m} \mu$, is a dimer. ALLEN ${ }^{23}$ and BUTLER ${ }^{24}$ actually observed the absorption of the $\mathrm{C}_{a 705}$ form.

At still longer wavelengths, around $735 \mathrm{~m} \mu$ (see refs. 25-30), absorption of a photomorphogenic pigment influencing photosynthesis was noticed, while weak bands were also observed at $75^{\circ}-760 \mathrm{~m} \mu$ (see ref. 3I). As the chemical structure of these pigments is still unknown, they will be left out of further consideration here.

Because of overlapping absorptions of various non-chlorophyllous pigments, the Soret band is hard to be studied. After finishing the present experiments, we learned that CEDERSTRAND AND GovindJEE ${ }^{32}$ attempted an examination of the blue region and obtained indication of a possible occurrence of five chlorophyll $a$ forms.

Of the red absorption band, $\mathrm{C}_{a} 680$ and $\mathrm{C}_{a} 670$ are the most predominant forms ${ }^{33,34}$. As a rule, the other forms show up much weaker than $C_{a} 670$. Moreover, even in leaves of one and the same plant the exact location of the maximum and the shoulders of the red absorption band may vary slightly. This may also be true of the number of the shoulders. As a consequence, it may be that part of the shoulders has escaped attention. Therefore, it seemed worth while to study the number, frequency, location, and some of the properties of the various chlorophyll $a$ forms, as evidenced by the structure of the red absorption band, at a large number of chloroplast preparations, from a single species. The results of this study are presented below.

\section{MATERIAL AND METHODS}

\section{Chloroplast preparation}

Leaves of Aspidistra elatior were subsequently rinsed with distilled water and cooled in $0.02 M$ phosphate buffer, $\mathrm{pH}$ 6.9. At $\mathrm{o}^{\circ}$ and in as weak light as possible, one leaf at a time was minced in a Braun multipress. The juice was taken up in the same buffer and filtered through filter paper. Next, at room temperature, the light 
absorption of the suspension was adjusted to about $70 \%$ at the maximum around $680 \mathrm{~m} \mu, \mathrm{I} \mathrm{cm}$ path length, by dilution with buffer.

When comparing spectra at different $\mathrm{pH}$, only distilled water was used during the preparation of the suspension. Samples of this suspension were mixed $\mathrm{I}$ : I with $0.04 M$ phosphate buffer of various $\mathrm{pH}$ 's.

\section{Heating}

The mentioned temperatures in the heat-experiments refer to the water bath in which sealed reagent tubes containing the chloroplast suspensions were placed for the given periods.

\section{Absorption measurements}

Absorption spectra were measured with a Beckman DK2 recording spectrophotometer, using I-cm cuvettes. For optimal results with the chloroplast suspension, a combination of slit widths, noise, and speed was selected. With the used instrument, this optimal combination was obtained by adjusting the dials as follows: $\lambda$ speed, 50 or 100 ; scale, $2 \times$; sensitivity, 3.14; and period, 0.6 or 2 . When measuring difference spectra in the $10 \%$ range, the dial positions were: 20 or $50,2 \times, 3.14$, and 0.6 or 2 respectively.

\section{Shape of the red band}

\section{RESULTS}

Fig. I shows an example of the red absorption band of Aspidistra chloroplasts. Apart from the main peak at $679 \mathrm{~m} \mu$, five weak shoulders are discernable. At first, part of such shoulders escaped our attention. However, after careful examination of

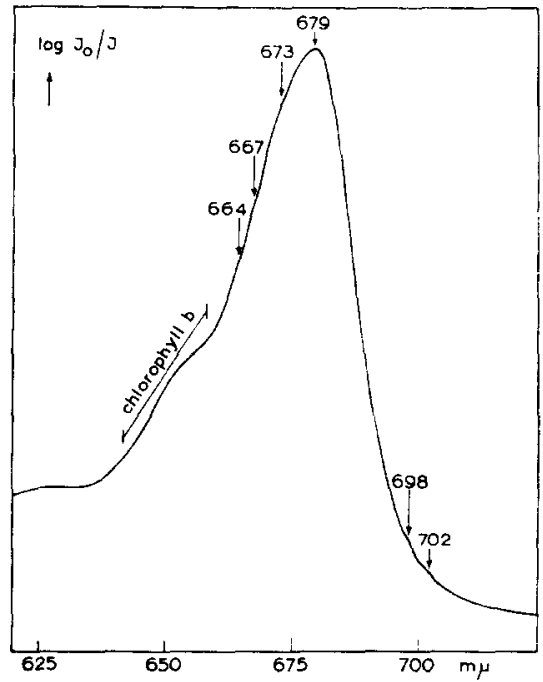

Fig. 1. Red absorption band of Aspidistra elatior chloroplasts.

a number of recordings it became clear that the weak irregularities did not occur casually. Moreover, they do not show up in recordings of transmission bands of G.A.B. interference filters in the same spectral region. Therefore, the weak shoulders, repeatedly observed in recordings with the same chloroplast preparation, cannot be 
instrumental artifacts. However, in addition to these shoulders irregularities, due to e.g. fluctuations of the mains or possible friction anomalies of the recorder pen may occur. In order to avoid misinterpretations in this respect, a duplicate recording with each sample was made. Only shoulders present in both recordings at the same wavelength were taken into account. In this way 50 chloroplast preparations from the same number of leaves of one and the same Aspidistra specimen, grown at moderate light intensities, were studied. Necessarily, the age of the leaves varied. However, all of the used leaves were full-grown.

It was found that the location of both the main peak and the shoulders, as well as the number of the latter varied slightly. Fig. I represents a selected recording which looks most "complete". At least, one shoulder around $670 \mathrm{~m} \mu$ was always observed. The frequency of peak and shoulders are plotted versus wave length in Fig. 2. The picture suggests that, apart from the main maximum around $678 \mathrm{~m} \mu$, five shoulders occur at least. They are located around $664,667,673,697$ and $703 \mathrm{~m} \mu$.

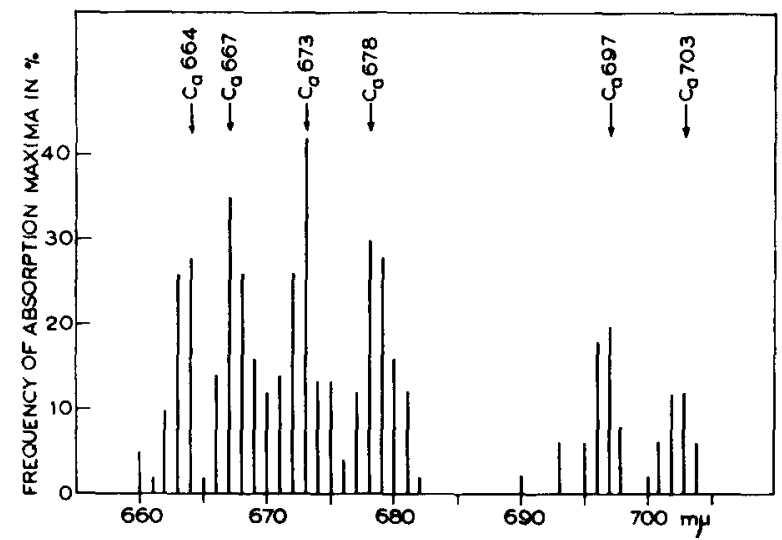

Fig. 2. Frequency of the occurrence of the components of the red absorption band in Aspidistra at different wavelengths.

The shoulders around 664,697 and $703 \mathrm{~m} \mu$ are less frequently observed than those around 667 and $673 \mathrm{~m} \mu$, while, of course, the main peak is always present. It seems that the location of the 667 and $673-\mathrm{m} \mu$ shoulders varies less than that of the main peak and the remaining shoulders. No indication of fixed combinations of shoulders was observed: all of them seem to occur independently of each other.

\section{Effect of $p H$}

In the range of 8 to 5 , the $\mathrm{pH}$ affects the red band only slightly (cf. Fig. 3). In between these limits, lowering of the $\mathrm{pH}$ may induce a very small shift of the band to longer wavelengths. Since scattering changes occur simultaneously, it is hard to conclude whether the I-m $\mu$ shift (Fig. 3) as well as the reduction of the significance of the weak shoulders is a true absorption change. In order to avoid changes in the pigment molecules, the studied $\mathrm{pH}$ range was kept relatively narrow.

\section{Effect of heating}

Fig. 4 shows heat-induced changes of the red absorption band. The heat-stability of components at $667 \mathrm{~m} \mu$ and shorter wavelengths is considerably higher than that 
of the components at longer wavelengths. Upon storage, the spectrum of a heated suspension changes only slightly. This indicates that the heat-induced effects are irreversible, and the formed products relatively stable.

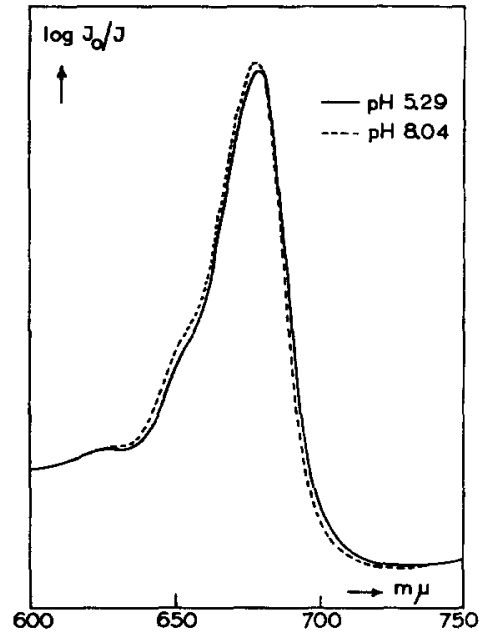

Fig. 3. Effect of $\mathrm{pH}$ on the red absorption band in Aspidistra.

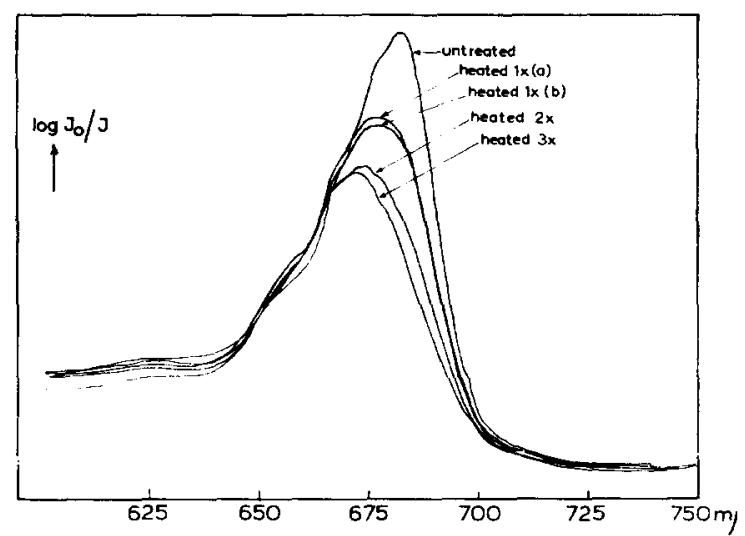

Fig. 4. Effect of heating on the red absorption band in Aspidistra. Meaning of indications on heating: IX(a): suspension heated at $65^{\circ}$ for heating: IX (a): suspension heated at $65^{\circ}$ for
$5 \mathrm{~min} ; \mathrm{IX}(\mathrm{b})$ : same suspension after $3 \mathrm{~h}$ storage at room temperature; $2 \mathrm{X}$ : same suspension ad-
ditionally heated for Io min at $65^{\circ} ; 3 \mathrm{x}$ : same suspension heated for 1o min at $65^{\circ}$ once more.

\section{Efect of storage}

Upon storage at room temperature, $2 \mathrm{I}-23^{\circ}$, slight alterations of the red band shape occur. These may be studied by examining difference spectra.

A spectral change results from either a variation of the pigment concentration or some alteration of pigment properties. In the first case, the difference spectrum is of the same shape and occurs at the same wavelengths as the absorption spectrum of the pigment. Depending on the sign of the concentration variation, it will be either negative or positive. In the second case, the resulting difference spectrum will resemble an oscillation recording, while the "phase" of the "oscillation" depends on the direction of the shift. In order to illustrate this effect a schematic absorption band, composed of three symmetrical bands of different shape is drawn, as well as "difference spectra" which result by shifting a varying number of components, cf. Fig. 5. It is clear that the number of "oscillations" depends on the amount of shifted components. However, an "oscillation" is symmetric only if no overlap of the bands of two or more shifted components occurs.

The wavelengths indicated in Fig. 5 are chosen to approximate the red absorption band of chlorophyll $a$ in vivo in a simplified way. The experimentally observed shift is about $\mathbf{I}-\mathbf{2} \mathbf{m} \boldsymbol{\mu}$. In order to demonstrate the effect of shift lengths over distances comparable with the experimentally observed ones, the difference spectra for shifts over both 2 and $4 \mathrm{~m} \mu$ are drawn. It is evident that the length of the shift is related to the "amplitude" of the "oscillation".

In order to study the "storage effect", a chloroplast preparation was divided 
into two samples one of which was stored at room temperature while the other one was kept at $0^{\circ}$. Both samples were kept in the dark. After I or $2 \mathrm{~h}$, the difference spectrum was recorded. The $o^{\circ}$-sample was placed at the "reference" position. Recording was started as soon as this sample had attained room temperature. An example out of a large number of difference-spectrum recordings is shown in Fig. 6. All recordings look similar.

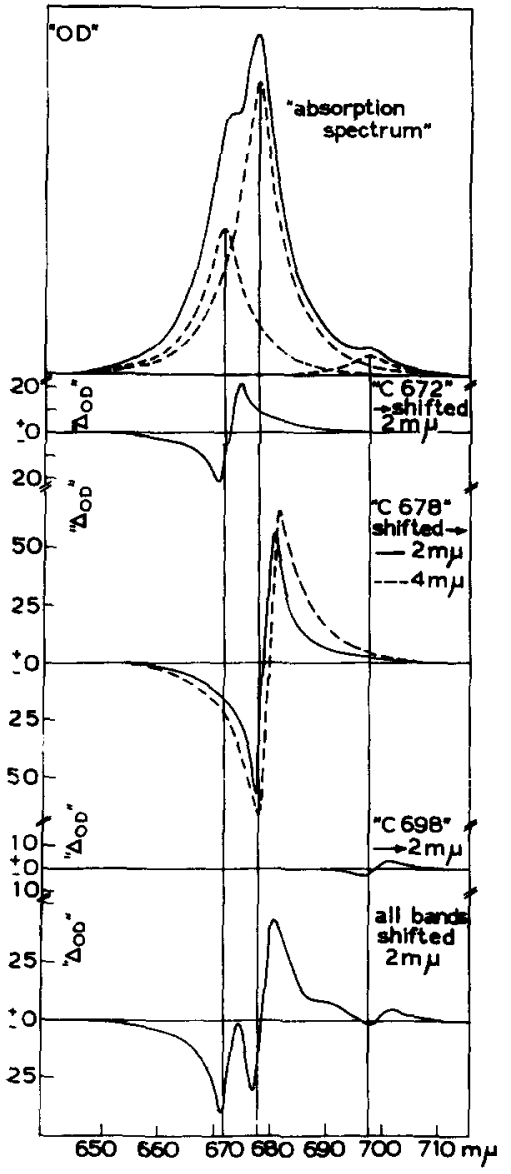

Fig. 5. Construction of a simplified absorption band of chlorophyll $a$, and difference spectra obtained by shifting the components both separately and combined. Moreover, the effect of shift length.

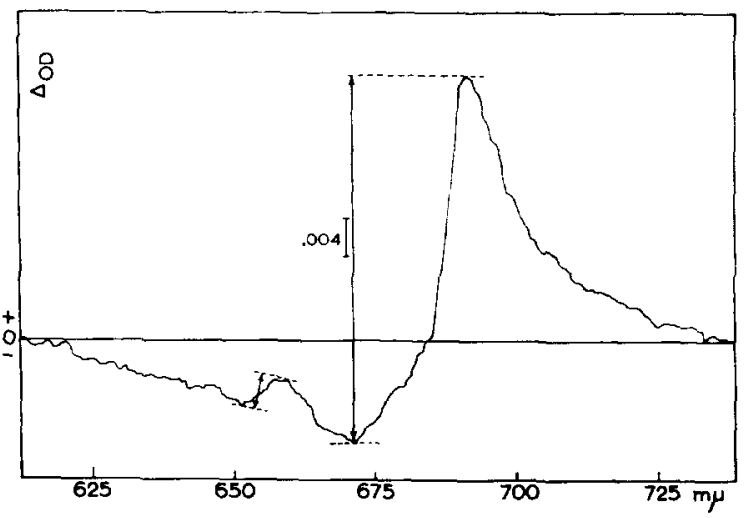

Fig. 6. Difference spectrum of two samples of $A$ spidistra chloroplast suspensions, stored in the dark for $2 \mathrm{~h}$, one of the samples at $0^{\circ}$, the other one at $23^{\circ}$. The " 0 " sample" was placed in the position of the "reference" cuvette in the spectrophotometer. The "amplitude" of the "oscillation" is taken as a measure for the extent of the spectral shift.

Two effects can be noticed: (a) a large "oscillation" probably due to a shift of the major absorption maximum around $678 \mathrm{~m} \mu$ (major shift), and (b) a much smaller "oscillation", due to a shift of a minor component (minor shift). It is hard to identify the component responsible for the latter shift. It may be chlorophyll $b$. However, it may as well be a short-wave form of chlorophyll $a$.

One remark remains to be made. The major shift does not yield a symmetric "oscillation". There may be three possible reasons for this phenomenon: first, the band of the shifted component is asymmetric; second, a number of minor components, spectroscopically located near to each other at the short-wave side of the complex absorption band, is shifted; and third, a combination of both shift and broadening of the band occurs. At present, any conclusion in this respect is refrained from. 


\section{Time course of the "storage effect"}

If the $0^{\circ}$-sample is kept in the spectrophotometer, it heats up and attains the same temperature as the sample stored at about $23^{\circ}$. The $0^{\circ}$-sample then starts to change and, thus, the spectroscopic differences between both samples decrease. As a measure for these differences, the "amplitude" of the "oscillations" (cf. Fig. 6) was taken and plotted versus time. From Fig. 7 it is evident that the time courses of the major and minor shifts are fundamentally different. As a rule, the first one needs $\mathrm{I}-2 \mathrm{~h}$ to run to completion, while this period amounts for the lat ter one only $20-40 \mathrm{~min}$.

\section{"Storage effect" and Hill reaction}

It is generally known that the ability of isolated chloroplasts to perform the Hill reaction with $1^{-2} M p$-benzoquinone as the oxidant is readily lost at room temperature. It therefore looked tempting to examine the time courses of both the spectroscopic change and the Hill-reaction rate at about $25^{\circ}$ and saturating light intensity with samples of the same chloroplast preparation simultaneously. 5 experiments, vielding the same results, were performed. An example is shown in Fig. 8 .

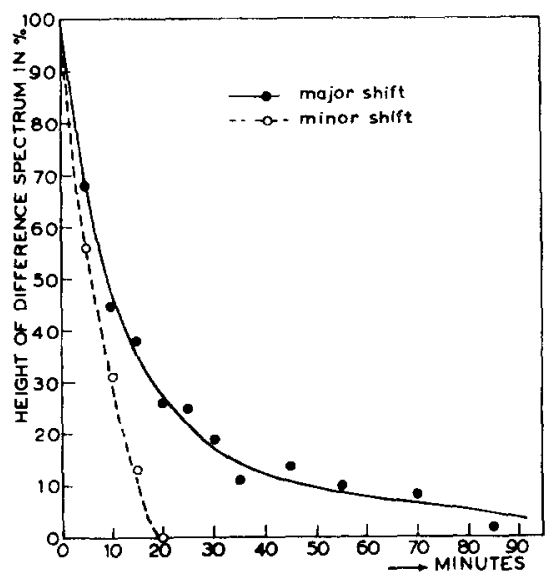

Fig. 7. Time courses of the "major" and "minor" shifts, as shown in Fig. 6. The value of the "oscillation amplitude" (see text) extrapolated to time zero was taken for $100 \%$.

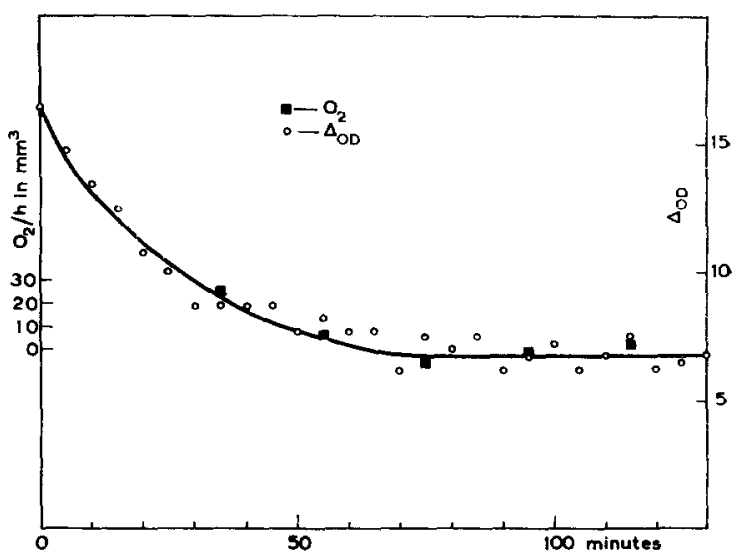

Fig. 8. Indication of coincidence of the time courses of the "major" absorption shift and the loss of ability to perform the Hill reaction, both of them at $25^{\circ}$. Hill reaction mixture: Warburg buffer No. 9 plus 1o ${ }^{-2} M p$-benzoquinone.

As temperature equilibration of the Warburg vessels in the thermostat required $25 \mathrm{~min}$, the major part of the photochemical activity was lost at the start of the experiment. Therefore, the present experiments only indicate that the photochemical oxygen liberation and the spectroscopic "major shift" come to a halt at about the same time. This indication is confirmed by the results of potentiometric experiments, made in collaboration with Dr. J. C. GoedHeER. With this method it is possible to start measurements earlier after raising the temperature. These and related experiments will be published at a later date.

\section{DISCUSSION}

Since the light scattering of its aqueous extracts is relatively weak, Aspidistra was chosen as a chloroplast source. At the same time, it is a disadvantage that both 
chlorophyll $a$ and $b$ occur in this object. Therefore, doubt may arise whether part of the short-wave shoulders should be ascribed to chlorophyll $a$ or $b$ complexes. However, the chlorophyll $b$ shoulder is quite smooth at both the short-wave and long-wave sides. It therefore seems likely that all shoulders belong to the chlorophyll $a$ group As mentioned before, no interrelation between the occurrence of the various shoulders mutually as well as between the shoulders and the main peak was observed. It therefore is suggested that the five shoulders and the main peak may represent six forms of chlorophyll $a$. Whether they always occur simultaneously, be it in amounts varying to such a degree that a number of them sometimes escape detection with the spectrophotometer used, or whether part of them are absent in various preparations, cannot be decided. If all forms were involved in different photochemical processes in photosynthesis, which would mean a quantum requirement of at least 6 , it seems reasonable to suppose that they are always present in Aspidistra chloroplasts, even if they cannot be observed with the used method. It may be possible as well that some more forms remained unobserved in the present investigation. The use of other techniques or instruments may solve this problem. In any case, the above mentioned results seem to suggest that, in Aspidistra, at least six forms of chlorophyll a may occur.

Halld ${ }^{33}$ suggests that the $\mathrm{C}_{a} 670$ and $\mathrm{C}_{a} 680$ forms may consist of different chlorophyll-protein complexes. The heat-induced effects, observed in the present study, then may be interpreted to mean that the heat-stability of the various chlorophyll a complexes differs mutually. The spectroscopic change of chloroplasts, when stored at room temperature, is studied with difference spectra, $c f$. Fig. 6. The "phase" of the "oscillation" shows that the changes consist of shifts towards the long-wave side.

The data in Fig. 7 suggest that the major shift, probably attributable to $\mathrm{C}_{\boldsymbol{a}} 678$, may proceed along a rectangular hyperbola, while the minor shift around $660 \mathrm{~m} \mu$ may run exponentially with time. In other words, the results suggest that the major shift may be due to a bimolecular reaction, while the minor one may be based on a monomolecular effect. This suggestion, however, is only given with the utmost restriction. It necessarily requires that the relation between "amplitude" of the "oscillation" and extent of the absorption shift does not deviate from linearity too much for small changes. Whether this holds for both forms, depends on the exact shape of their absorption bands as well as the invariability of the band widths when shifting. At present, these prerequisites are not accessible for checking.

Preliminary experiments indicate that the ability to perform the Hill reaction with Io $^{-2} M$ quinone is lost as soon as the major shift is completed. The coincidence may suggest a relationship between this photochemical ability and the presence of the original $\mathrm{C}_{n} 678$ form. A study of this phenomenon is in progress.

\section{ACKNOWLEDGEMENT}

Thanks are due to Miss A. W. RHEEBERGEN for her skillful assistance in measuring the Hill reaction rates.

\section{REFERENCES}

${ }^{1}$ L. N. M. Duysens, Doctoral Thesis, State University, Utrecht, $195^{2}$.

C. S. Yocum AND L. R. Blinks, J. Gen. Physiol., 38 (I954) I.

a C. S. French and V. M. K. Young, in A. Hollaender, Radiation Biology, Vol. 3, McGrawHill Book Co., New York, Toronto, London, 19.56, p. 343. 
4 F. Haxo and L. R. Blinks, J. Gen. Physiol., 33 (1950) $3^{89 .}$

5 V. M. VOROB'Eva AND A. A. KRASNOvSKY, Biokhymia, 2 I (1956) 126.

- R. Emerson, R. Chalmers and C. Cederstrand, Proc. Natl. Acad. Sci. U.S., 45 (1959) 1703.

7 J. Franck, Proc. Natl. Acad. Sci. U.S., 44 (1958) 94 I.

* E. Rabinowitch, Discussions Faraday Soc., 1959, p. I6r.

- Govindjee and E. Rabinowitch, Science, 132 (I960) 355.

10 R. Govindjee, J. B. Thomas and E. Rabinowitch, Science, I32 (I960) 421 .

11 L. N. M. Duysens, J. Amesz and B. M. Kamp, Nature, I9o (196I) 42 I.

12 M. Losada, F. R. Whatley and D. I. Arnon, Nature, 190 (196I) 606.

13 H. T. Witt, A. Muller and B. Rumberg, Nature, I9I (I96I) I94.

14 C. S. French, Proc. Igth Ann. Biol. Coll., Oregon State College, 1958, p. 52.

15 C. S. FRE NCH, Brookhaven Symposia Biol., I I (1958) 65.

16 C. S. French, in W. D. McElroy and B. Glass, Light and Life, Johns Hopkins Press, 196 r, p. 447

17 C. S. French and D. C. Fork, Preprint Int. Biochem. Congr. Moscow, I96I.

18 B. Kox ANd G. Hoch, in W. D. McElroy AND B. Glass, Light and Life, Johns Hopkins Press, I 96 I; p. 397.

19 B. KoK, Biochim. Biophys. Acta, 48 (I96I) 527 .

20 A. A. Krasnovsky and L. M. Kosobutskaja, Doklady Acad. Nauk S.S.S.R., 9 I (19.53) 343.

21 S. S. BRODY, Science, 128 (1958) 838 .

$22 \mathrm{~S}$. S. Brody and M. Brody, Nature, i 89 (Igt I) 547.

${ }_{23}$ M. B. Allen, in W. D. McElroy and B. Glass, Light and Life, Johns Hopkins Press, r96!, p. 479 .

24 W. L. Butle R, Arch. Biochem. Biophys., 93 (196I) 4 I 3

25 W. L. Butler AND K. H. Norris, Arch. Biochem. Biophys., 87 (1960) 31 .

26 W. L. Butler, K. H. Norris, H. W. Siegelman and S. B. Hendricks, Proc. Natl. Acad. Sci. U.S., 45 (1959) 1703 .

37 J. B. Thomas and Govindjee; in W. D. McElroy ann B. Glass, Light and Life, Johns Hopkins Press, Ig6I, p. 475 .

28 J. B. Thomas and Govind JeE, Biophys. J., I (1960) 63

28 E. Rabinowitch, Govindjee and J. B. Thomas, Science, $132(1960) 422$.

30 Govindjee, E. Rabinowitch and J. B. Thomas, Biophys. J.. I (Ig6o) 9 I.

31 Govindjee, C. Cederstrand and E. Rabinowitch, Science, I 34 (ro6 I) 391.

32 C. CeDerstrand and GovindjeE, personal communication.

33 P. Halldal, Physiologia Plantarum, I I (1958) 401.

34 J. B. Thomas and J. W. Marsman, Biochim. Biophys. Acta, 35 (1959) 310.

Biochim. Biophys. Acta, 59 (1962) 202-210 